\title{
Rapid Assessment of Agents of Biological Terrorism: Defining the Differential Diagnosis of Inhalational Anthrax Using Electronic Communication in a Practice- Based Research Network
}

Jonathan L. Temte, $M D, P b D^{1,2}$

Anna Lisa Anderson, BS ${ }^{3}$

${ }^{1}$ Wisconsin Research Network, Madison, Wis

${ }^{2}$ Department of Family Medicine, University of Wisconsin, Madison, Wis

${ }^{3}$ University of Wisconsin Medical School, Madison, Wis

\begin{abstract}
PURPOSE Early detection of bioterrorism requires assessment of diagnoses assigned to cases of rare diseases with which clinicians have little experience. In this study, we evaluated the process of defining the differential diagnosis for inhalational anthrax using electronic communication within a practice-based research network (PBRN) and compared the results with those obtained from a nationwide random sample of family physicians with a mailed instrument.
\end{abstract}

\begin{abstract}
METHODS We distributed survey instruments by e-mail to 55 physician members of the Wisconsin Research Network (WReN), a regional PBRN. The instruments consisted of 3 case vignettes randomly drawn from a set describing 11 patients with inhalational anthrax, 2 with influenza $A$, and 1 with Legionella pneumonia. Physicians provided their most likely nonanthrax diagnosis, along with their responses to 4 yes-or-no management questions for each case. Physicians who had not responded at 1 week received a second e-mail with the survey instrument. The comparison group consisted of the nationwide sample of physicians who completed mailed survey instruments. Primary outcome measures were response rate, median response time, and frequencies of diagnostic categories assigned to cases of inhalational anthrax.
\end{abstract}

RESULTS The PBRN response rate compared favorably with that of the national sample (47.3\% vs $37.0 \%$; $P=$ not significant). The median response time for the PBRN was significantly shorter than that for the national sample (2 vs 28 days; $P$ $<.001)$. No significant differences were found between the PBRN and the Midwest subset of the national sample in the frequencies of major diagnostic categories or in case management.

CONCLUSIONS Electronic means of creating differential diagnoses for rare infectious diseases of national significance is feasible within PBRNs. Information is much more rapidly acquired and is consistent with that obtained by conventional methods.

Ann Fam Med 2004;2:434-437. DOI: 10.1370/afm.120.

\section{INTRODUCTION}

Tarly detection of a bioterrorism event may depend on the recognition -1 of clusters of unusual cases or patterns of acute illnesses. To this end, major efforts are currently directed toward electronic surveillance of large populations using routinely collected information from patient encounters, such as International Classification of Diseases, Ninth Revision (ICD-9) codes or a combination of codes. ${ }^{1-3}$ Such information, however, 
may be difficult to interpret if the underlying behavior of primary care physicians is not well defined.

Clinicians rarely arrive at a definitive diagnosis when they first examine a patient. Rather, they combine symptoms, patient history, findings of physical and laboratory examination, and their own experience to develop a rational possibility for a diagnosis, which may, in turn, be entered onto a billing form. ${ }^{4}$ The complete set of rational diagnoses for a given set of information is known as the differential diagnosis.

In an age of bioterrorism and globally emerging infectious diseases, a critical element of biodefense is the ability to define rapidly the primary care differential diagnosis of a rare and unfamiliar disease. The set of diagnoses and their individual probabilities may then be used to better inform the development of surveillance systems.

In a related study, a primary care differential diagnosis of inhalational anthrax was developed by mailing clinical case vignettes to a nationwide random sample of family physicians who were active members of the American Academy of Family Physicians (AAFP). ${ }^{5}$ This approach was laborious and did not have the extreme timeliness required for rapidly emerging diseases. This study assesses the feasibility, timeliness, and validity of using similar case vignette methods enhanced by electronic distribution to a limited, nonrandom set of physicians within a regional practice-based research network (PBRN).

\section{METHODS}

The Wisconsin Research Network (WReN) is a long-standing, regional PBRN. Its family physician members are located within Wisconsin. The 60 current members were given the opportunity to opt out of this study, and 5 chose not to participate.

We used 11 clinical case vignettes of inhalational anthrax that have been described elsewhere, ${ }^{5-7}$ as well as 2 cases of influenza and 1 case of Legionella pneumonia. From these 14 cases, 3 were randomly selected and sent by e-mail in random order to each participant along with a cover letter describing the study.
A second e-mail was sent to nonrespondents 7 days after the initial e-mail.

After evaluating each vignette, WReN physicians were instructed to provide their most likely nonanthrax diagnosis and to respond to 4 management options: (1) hospitalize the patient, (2) obtain a chest radiograph at their clinic, (3) obtain a specimen for blood culture at their clinic, and (4) initiate empiric antibiotic therapy at their clinic. They returned their responses by reply e-mail.

We compared the results obtained from the WReN family physicians by e-mail with those obtained from the previously mentioned nationwide AAFP cohort surveyed by conventional mail. ${ }^{5}$ Response rates were compared using the chi-square test. Median response times were compared using the Kruskal-Wallis statistic. When comparing the results for diagnoses and case management, we used the Midwest subset of the AAFP survey (101 physicians) as a comparison group to account for possible regional differences in diagnoses and management decisions. We placed diagnoses for the cases of inhalational anthrax into 6 broader diagnostic categories: pneumonia, influenza/bronchitis, central nervous system

\section{Table 1. Comparison of Survey Methods, Response Rates and Times, and Hypothetical Case Management Approaches}

\begin{tabular}{|c|c|c|c|}
\hline Characteristic & AAFP Survey 5 & WReN Survey & $P$ Value \\
\hline \multicolumn{4}{|l|}{ Survey method } \\
\hline Location & Nationwide & Wisconsin & \\
\hline Physicians eligible, No. & 33,365 & 60 & \\
\hline $\begin{array}{l}\text { Physicians sent survey } \\
\text { instruments, No. }\end{array}$ & 662 & 55 & \\
\hline Method of survey distribution & $\begin{array}{l}\text { Sent by conventional } \\
\text { mail with self- } \\
\text { addressed, stamped } \\
\text { response postcard }\end{array}$ & $\begin{array}{l}\text { Sent by e-mail with } \\
\text { instructions to } \\
\text { reply by e-mail }\end{array}$ & \\
\hline Distributions, No. & 3 & 2 & \\
\hline Time between distributions, days & 21 & 7 & \\
\hline Direct cost per response, $\$$ * & 5.59 & Nominal & \\
\hline \multicolumn{4}{|l|}{ Response rate and time } \\
\hline Physicians responding, No. (\%) & $245(37.0)$ & $26(47.3)$ & NS \\
\hline Range of response times, days & $7-172$ & $1-21$ & \\
\hline Median response time, days & 28 & 2 & $<.0001$ \\
\hline \multicolumn{4}{|l|}{ Case management ${ }^{\dagger}$} \\
\hline Hospitalize the patient, \% & 62.9 & 62.7 & NS \\
\hline Obtain a chest radiograph, \% & 84.2 & 86.7 & NS \\
\hline $\begin{array}{l}\text { Obtain a specimen for blood } \\
\text { culture, \% }\end{array}$ & 55.2 & 45.9 & NS \\
\hline $\begin{array}{l}\text { Initiate empiric antibiotic } \\
\text { therapy, \% }\end{array}$ & 58.8 & 45.9 & NS \\
\hline \multicolumn{4}{|c|}{$\begin{array}{l}\text { * Total cost of printing, envelopes, and postage divided by number of responses. The associated personnel costs } \\
\text { are not included. } \\
\text { † For case management, we compared responses between the Midwest subset of the AAFP sample ( } 101 \text { physicians } \\
43 \text { respondents, and } 97 \text { responses to cases of inhalational anthrax evaluated) and WReN physicians ( } 62 \text { responses } \\
\text { to cases of inhalational anthrax evaluated). Values are percentages of cases for which respondents indicated that } \\
\text { they would use that management strategy. }\end{array}$} \\
\hline
\end{tabular}


Figure 1. Accumulation of responses over time.

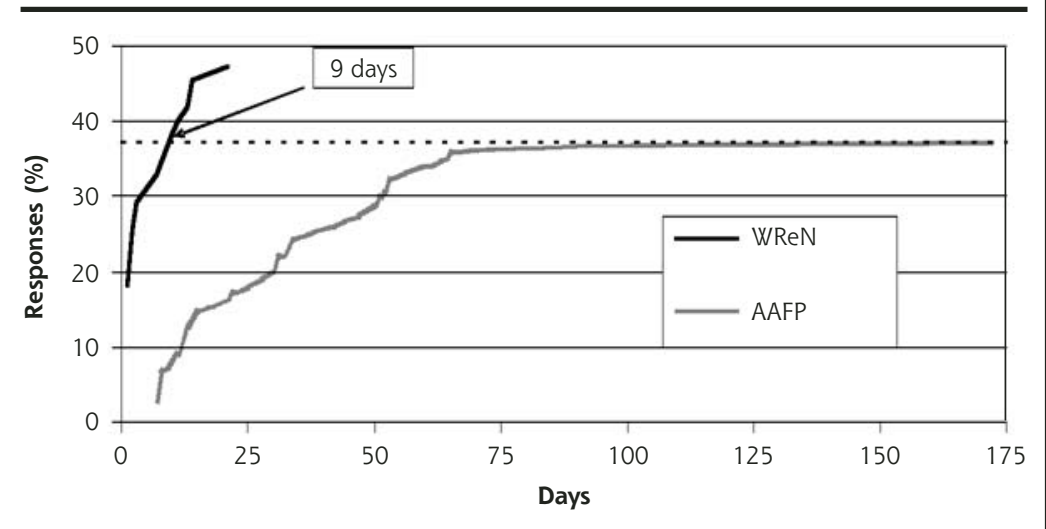

WReN = Wisconsin Research Network; AAFP - American Academy of Family Physicians.

Figure 2. Distributions of diagnostic categories assigned to cases of inhalational anthrax.

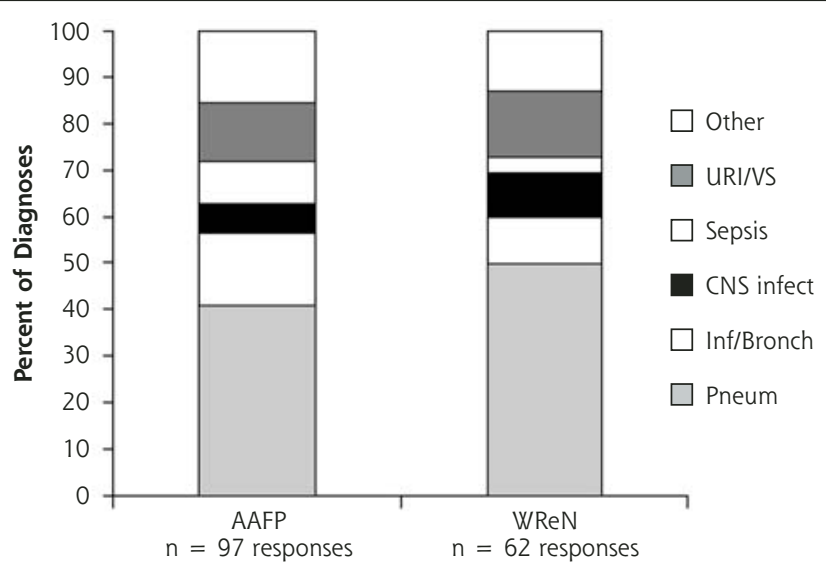

AAFP $=$ American Academy of Family Physicians; W/ReN = Wisconsin Research Network; URI/VS = upper respiratory tract infection/viral syndrome; CNS infect $=$ central nervous system infection; Inf/Bronch $=$ influenza/bronchitis; Pneum $=$ pneumonia. contact nonrespondents in the former group (Table 1). This difference, however, was not statistically significant. The PBRN attained the final AAFP response rate on day 9 (Figure 1). The median response time for WReN respondents was 2 days (Table 1). This timeliness was 14-fold faster than that in the AAFP survey group (Kruskal-Wallis test; $\mathrm{H}=48.60, d f=1, P<.001)$.

WReN physicians provided 62 responses to the cases of inhalational anthrax and assigned 18 unique diagnoses; 15 responses to the nonanthrax cases were not used. The Midwest subset of AAFP respondents provided 97 responses to the cases of inhalational anthrax. We found no significant differences in the relative frequencies of 6 diagnostic categories assigned to cases of inhalational anthrax among WReN and Midwest AAFP respondents (Figure 2; $\left.\chi^{2}=4.53, d f=5, P=.477\right)$.

The hypothetical initial management of the inhalational anthrax cases did not differ between the WReN physicians and the Midwest AAFP cohort. Rates of hospitalization and obtaining chest radiographs were nearly identical (Table 1 ). WReN physicians were slightly less likely to obtain specimens for blood culture and to start empiric antibiotic therapy in the outpatient setting. infections, sepsis, upper respiratory tract infection/viral syndrome, and other. The frequencies of responses in each category between survey method groups were compared using the chi-square test. Finally, initial management decisions between the survey method groups were compared using the chi-square test.

The University of Wisconsin Medical School Institutional Review Board approved the study, and we obtained appropriate informed consent according to the approved protocol.

\section{RESULTS}

The response rate from the WReN group was $10 \%$ greater than that from the nationwide AAFP survey group even though fewer attempts were made to

\section{DISCUSSION}

This small study illustrates 3 primary findings related to rapid assessments for bioterrorism and emerging infections. First, response rates from electronically based PBRN studies are at least as good as those from traditional surveys using survey instruments mailed to a larger population. Second, the timeliness of response is greatly enhanced using electronic communication within PBRNs. The 14 -fold reduction in the median response time is especially noteworthy for situations demanding extreme timeliness of response. ${ }^{1}$ Third, the validity of results emerging from a PBRN for the diagnostic spectrum and hypothetical initial management of inhalational anthrax cases appears quite good. ${ }^{8,9} \mathrm{We}$ noted no significant differences on a gross scale. 
Full comparisons were not possible because of the narrow scope of this study. The PBRN response rate was limited because we sent 1 fewer request to nonrespondents relative to the AAFP survey, and because we deployed the electronic survey on June 30, thus overlapping with summer vacation schedules. The small sample size also precluded detection of subtle differences in the differential diagnosis, case management, or both.

An unexpected finding is noteworthy. Use of an electronic system for this study resulted in 8 documented e-mail delivery errors (ie, e-mail returned to the sender; rate $=8 \%$ ) and at least 4 documented reply failures (ie, the respondent could not submit a response; rate $\geq 15.4 \%$ ). Additional, undetected reply errors could have occurred, resulting in the respondent giving up. Future studies need to address the reliance on multiple e-mail services to widespread PBRN members and the presence of so-called firewalls that may block e-mail.

This small study clearly demonstrates the usefulness of PBRNs to gather data rapidly on time-sensitive topics. The potential benefits of nationwide primary care PBRNs and cooperatives of regional PBRNs in conducting similar studies on bioterrorism and emerging infections cannot be understated.

To read or post commentaries in response to this article, see it online at http://www.annfammed.org/cgi/content/full/2/5/434.

Key words: Bioterrorism; primary health care; anthrax; diagnosis, differential; electronic mail; practice-based research networks; computer communication networks

Submitted November 14, 2003; submitted, revised, January 8, 2004; accepted January 12, 2004.
Funding support: The University of Wisconsin Department of Family Medicine provided a stipend to Ms. Anderson through the 2003 Summer Student Research and Clinical Assistantship Program. Partial support was provided for this project by grant 1 R21-HS1349-01 from the Agency for Healthcare Research and Quality.

\section{References}

1. Wagner MM, Tsui F-C, Espino JU, et al. The emerging science of very early detection of disease outbreaks. J Pub Health Management Pract. 2001;7:51-59.

2. Lazarus R, Kleinman K, Dashevsky I, et al. Use of automated ambulatory-care encounter records for detection of acute illness clusters, including potential bioterrorism events. Emerg Infect Dis. 2002;8:753-760.

3. Lober WB, Karras BT, Wagner MM, et al. Roundtable of bioterrorism detection. Information system-based surveillance. J Am Med Informatics Assoc. 2002;9:97-104.

4. Okkes IM, Oskam SK, Lamberts H. The probability of specific diagnoses for patient presenting with common symptoms to Dutch family physicians. J Fam Pract. 2002;51:31-36.

5. Temte JL, Zinkle AR. The primary care differential diagnosis of inhalational anthrax. Ann Fam Med. 2004;2:438-444.

6. Jernigan JA, Stephens DS, Ashford DA, et al, and members of the Anthrax Bioterrorism Investigation Team. Bioterrorism-related inhalational anthrax: the first 10 cases reported in the United States. Emerg Infect Dis. 2001;7:933-944.

7. Barakat LA, Quentzel HL, Jernigan JA, et al, for the Anthrax Bioterrorism Investigation Team. Fatal inhalational anthrax in a 94-year-old Connecticut woman. JAMA. 2002;287:863-868.

8. Croughhan MS. Are the results of practice-based research generalizable? In: Methods for Practice-Based Research Networks. Challenges and Opportunities. Proceedings from the Practice-Based Research Networks Methods Conference. San Antonio, Texas, November 29-30, 2001. AAFP, Leawood, KS; 2001.

9. Nutting PA, Baier M, Werner JJ, Cutter G, Reed FM, Oranzo AJ. Practice patterns of family physicians in practice-based research networks: a report from ASPN. Ambulatory Sentinel Practice Network. J Am Board Fam Pract. 1999; 12:278-284. 